\title{
OCORRÊNCIA DE CÂNCER NA PRIMEIRA INFÂNCIA EM SANTA CATARINA: ANÁLISE DOS REGISTROS HOSPITALARES
}

\section{OCCURRENCE OF CANCER IN EARLY CHILDHOOD IN SANTA CATARINA: ANALYSIS OF HOSPITAL RECORDS}

\author{
Jane Kelly Oliveira Friestino \\ Universidade Federal da Fronteira Sul campus Chapecó, Chapecó, SC, Brasil \\ Universidade Estadual de Campinas, Faculdade de Ciências Médicas, Campinas, SP, Brasil \\ jane.friestino@uffs.edu.br \\ Rivaldo Mauro Faria \\ Universidade Federal de Uberlândia, Instituto de Geografia, Uberlândia, MG, Brasil \\ rivaldofaria.geo@gmail.com \\ Paulo Henrique de Araújo Guerra \\ Universidade Federal da Fronteira Sul campus Chapecó, Chapecó, SC, Brasil \\ paulo.guerra@uffs.edu.br
}

Priscila Maria Stolses Bergamo Francisco Universidade Estadual de Campinas, Faculdade de Ciências Médicas, Campinas, SP, Brasil primaria@unicamp.br

\begin{abstract}
RESUMO
A rede de atenção oncológica deve ser norteada a partir de indicadores e de características próprias do território, fomentando a oferta de serviços condizentes com a realidade. Com isso, objetivou-se identificar a tendência temporal e os locais de atendimento de câncer na primeira infância, segundo macrorregiões do estado Santa Catarina, entre os anos de 2009 e 2019. Trata-se de um estudo ecológico exploratório, descritivo, de natureza documental, com análise de registros hospitalares de câncer em crianças de 0 a 05 anos, atendidos na rede hospitalar de Santa Catarina entre 2009 a 2019. Foram identificados 1.030 registros hospitalares de câncer em crianças com idade $\leq 05$ anos no período estudado. A rede assistencial oncológica na primeira infância encontra-se concentrada nas macrorregiões de saúde da Grande Florianópolis e Planalto Norte e Nordeste, sendo estas as regiões mais populosas. Observou-se diminuição das ocorrências nas macrorregiões Grande Florianópolis e Planalto Norte e Nordeste, com importante aumento da macrorregião do Grande Oeste entre 2009 e 2019. Houve uma interiorização dos atendimentos ao longo dos anos, beneficiando regiões que se encontram mais distantes da maioria dos serviços de alta complexidade em oncologia pediátrica.
\end{abstract}

Palavras-chave: Assistência integral à saúde. Serviço hospitalar de oncologia. Mapeamento geográfico.

\begin{abstract}
The oncology care network would guided by health indicators and characteristics specific to the territory, promoting the provision of services based on reality. The objective is to produce and provide information about hospital care for cancer in early childhood, in the state of Santa Catarina. This is an exploratory, descriptive, documental ecological study, with analysis of hospital cancer records of children under 05 years of age, attended in the hospital network of Santa Catarina between 2009 and 2019. A total of 1,030 hospitalizations for cancer in children aged under 05 years were identified during the study period. The oncology care network of early childhood is concentrated in the health macro-regions of Grande Florianópolis and Planalto Norte e Nordeste, which are the most populous regions. There was a decrease in hospitalizations in the Grande Florianópolis and Planalto Norte e Nordeste macro-regions, with a significant increase in the Grande Oeste macro-region between 2009 and 2019. There
\end{abstract}

Recebido em: 13/01/2022

Aceito para publicação em: 27/01/2022.

\begin{tabular}{llllll}
\hline Hygeia & Uberlândia - MG & Edição especial: X GeoSaude & Fev./2022 & p. 110-119 & Página 110
\end{tabular}


has been an attended of care over the years, benefiting regions that are farther away from most high-complexity pediatric oncology services.

Keywords: Comprehensive health care. Oncology service, hospital. Geographic mapping.

\section{INTRODUÇÃo}

A primeira infância, caracterizada pelo período compreendido desde o nascimento até os cinco anos de idade, é tida como uma importante janela de oportunidades para o desenvolvimento integral de uma criança, de modo a construir uma base para o seu futuro (BLACK et al, 2017). O Fundo de Emergência Internacional das Nações Unidas para a Infância (UNICEF) recomenda o debate e a implementação de planos, ações e políticas públicas ampliadas que levem em conta os contextos, modos de vida, os cuidados de saúde e nutrição, proteção contra danos e sensação de segurança, tal como a oferta de oportunidades de aprendizagem precoce, para que todas as crianças possam aproveitar igualmente dessa janela de oportunidades (UNICEF, 2017).

No âmbito da saúde, mais especificamente, destaca-se a importância do monitoramento do processo de cuidado nas diversas dimensões da saúde infantil. O indicador da mortalidade infantil tem sido um indicador associado à melhoria das condições de vida e ao maior acesso das pessoas aos serviços de saúde, que são fatores precipuamente relacionados ao território e ao lugar (ALMEIDA; SZWARCWALD, 2012; ANDRADE; BASTOS, 2002; GOLDANI et al, 2001). Por essa razão, políticas de base territorial, sejam elas transversais (educação, moradia, renda, etc.), ou ligadas ao sistema de atenção, como a descentralização dos serviços, tiveram enorme impacto na redução da mortalidade infantil no Brasil. Entretanto, faltam ainda estudos geográficos mais amplos e sistemáticos para avaliar as condições territoriais de indicadores, embora avanços tenham sido feitos nos últimos anos (ALMEIDA; SZWARCWALD, 2012; DIAS et al, 2019).

De maneira geral, são ainda fragmentados os estudos dos indicadores de saúde infantil no Brasil. Há inúmeros esforços na identificação das razões sociais, assistenciais ou geográficas do óbito infantil, mas ainda é necessário fortalecimento no sentido de se produzir um índice composto que permita discutir a saúde infantil e o desenvolvimento infantil numa perspectiva ampla. É o caso, entre outros, do Índice de Saúde Infantil proposto pela UNICEF (2008) para medir o estado da saúde infantil e as condições de seu desenvolvimento.

As múltiplas dimensões da saúde infantil exigem também estudos agregados de indicadores. Efetivamente, há duas dimensões da saúde infantil que precisam ser pensadas conjuntamente pela geografia (FARIA; SANTANA, 2015): i) os resultados em saúde, representados pelos indicadores de mortalidade e morbidade; ii) os determinantes em saúde, representados pelos indicadores médicoassistenciais; demográficos, biológicos e sócio-territoriais. Com isso, os perfis territoriais da saúde infantil irão expressar essas duas dimensões, não apenas como resultado do território, mas como expressão da própria existência territorial, como um dado vivo e atual (FARIA, 2013). Porém, é ainda fundamental avaliar situações específicas, não apenas como indicadores compostos, mas como variáveis individuais que concorrem para produzir condições muito próprias de vida e saúde infantil nos territórios, como o caso do câncer infantil, ou seja, uma variável individual extremamente importante para redução da mortalidade infantil no Brasil.

Na temática do câncer infantil, é considerável a produção de dados para apoiar a reorganização do Sistema Único de Saúde (SUS), ora em relação à oferta do adequado atendimento e cuidado, em vista das necessidades específicas das crianças, ora pelo fortalecimento dos cenários que ofertam distintos níveis de atenção. Por outro lado, esses dados também permitem o reconhecimento da identidade regional que, por sua vez, se constitui como um dos elementos fundamentais para a organização da regionalização dos serviços de saúde (DORNELES; AREOSA, 2020). De fato, a ocorrência do câncer, nomeadamente na primeira infância, aponta para necessidade de ações programáticas de planejamento territorial, sobretudo do planejamento baseado em políticas de pactuações, como é caso do SUS (BRASIL, 2006), as quais exigem instrumentos de avaliação amplos, consistentes e abrangentes, capazes de integrar a natureza complexa dos problemas. Um dos elementos fundamentais para a organização da regionalização.

A oncologia pediátrica apresenta especificidades importantes, ao mesmo tempo em que os casos são considerados uma ocorrência rara, estes representam a principal causa de morte por doença em crianças no Brasil, denotando a relevância em possuir uma rede de atenção que valorize tanto o diagnóstico precoce, quanto o tratamento efetivo (PIRES, 2018).

\begin{tabular}{llllll}
\hline Hygeia & Uberlândia - MG & Edição especial: X GeoSaude & Fev./2022 & p. 110-119 Página 111
\end{tabular}


O conhecimento sobre a distribuição de atendimentos hospitalares de crianças com câncer em um determinado território pode apoiar a formulação de políticas públicas em vista de melhorar as condições de vida dos pacientes e seus familiares. Isso porque, conhecer a dinâmica de atendimentos auxilia na formulação de estratégias e desenvolvimento das redes de atenção oncológica, pois o tratamento para essas enfermidades possui grande impacto e exige uma boa articulação entre os serviços, incluindo desde a Atenção Primária até os centros especializados. Sendo assim, foi objetivo do presente estudo apresentar o perfil dos atendimentos hospitalares de câncer ocorridos na primeira infância, no estado Santa Catarina.

\section{METODOLOGIA}

O presente trabalho constitui-se como um recorte oriundo de uma pesquisa maior intitulada: Índice de Saúde Infantil: avaliação multicritério dos principais resultados e determinantes da saúde infantil no Brasil, a qual tem sido desenvolvida inicialmente nos estados de Santa Catarina e Rio Grande do Sul. Trata-se de um estudo ecológico exploratório, descritivo, de natureza documental, que utilizou dados secundários dos registros hospitalares de câncer ocorridos na primeira infância, 0 a 05 anos de idade, atendidos na rede hospitalar do estado de Santa Catarina, no período entre 2009 e 2019.

No presente estudo foram considerados somente os hospitais habilitados pelo Ministério da Saúde como unidades de alta complexidade em oncologia, classificados como Centros de Assistência de Alta Complexidade em Oncologia (CACON) e Unidades de Assistência de Alta Complexidade (UNACON), pois nestes serviços há a presença dos Registros Hospitalares de Câncer (RHC), que agrupam informações detalhadas a respeito da terapêutica das pessoas com câncer. A coleta dos dados foi realizada por meio do Integrador dos Registros Hospitalares de Câncer (RHC), disponibilizado pelo Instituto Nacional de Câncer (INCA), que é o órgão nacional que unifica e auxilia as unidades de atendimento de alta complexidade em oncologia a realizarem seus registros, seguindo padrões internacionais, normatizados pela IACR - International Association of Cancer Registries. O Integrador dos Registros pode ser acessado de forma livre via Tabnet (INCA, 2021).

Para o levantamento foram consideradas as variáveis: número de casos de câncer na primeira infância, por ano da primeira consulta, procedência e unidade de atendimento, durante os anos 2009 e 2019. As unidades hospitalares de atendimento foram identificadas por meio dos tabuladores do RHC, e o município ao qual pertenciam bem como o nome, foram consultados no Cadastro Nacional dos Estabelecimentos de Saúde (CNES). As unidades hospitalares foram agrupadas de acordo com o município de localização e os mesmos foram alocados nas 07 macrorregiões de saúde descritas no Plano Diretor de Regionalização (PDR) do estado de Santa Catarina (SANTA CATARINA, 2018). Desta forma, foram obtidos o número de registros hospitalares no período para compreensão do perfil temporal (série histórica) e de distribuição espaço-geográfica, de dados hospitalares de crianças em tratamento oncológico. A coleta de dados foi realizada no mês de abril de 2021.

Os dados foram armazenados e tabulados em planilhas eletrônicas. Estes foram analisados por meio de frequências absolutas e relativas, bem como análise de correlação entre número absoluto e a distribuição anual dos registros hospitalares no período de 2009 a 2019. Foi utilizado o coeficiente de correlação de Spearman considerando-se significativo o valor de $p<0,05$. Para as análises e apresentação dos resultados foram utilizadas planilhas eletrônicas do Google, o software Epidata e Quantum Gis, todos de livre acesso. Por tratar-se de dados públicos, não se fez necessária apreciação da proposta em comitê de ética e pesquisa.

\section{RESULTADOS}

Foram identificados 1.030 registros hospitalares de câncer em crianças menores de cinco anos de idade entre os anos 2009 e 2019 em Santa Catarina. Verificou-se que a maioria das ocorrências se deu na macrorregião da Grande Florianópolis (59\%), seguida pela região Planalto Norte e Nordeste $(25 \%)$. A macrorregião com o menor número de ocorrência de câncer na primeira infância foi a de Foz do Itajaí $(0,6 \%)$, conforme descrito na Figura 1.

\begin{tabular}{llllll}
\hline Hygeia & Uberlândia - MG $\quad$ Edição especial: X GeoSaude & Fev./2022 & p. 110-119 & Página 112
\end{tabular}


Figura 1. Distribuição dos registros hospitalares de câncer em menores de cinco anos de idade, de acordo com a macrorregião de Saúde. Santa Catarina. 2009-2019

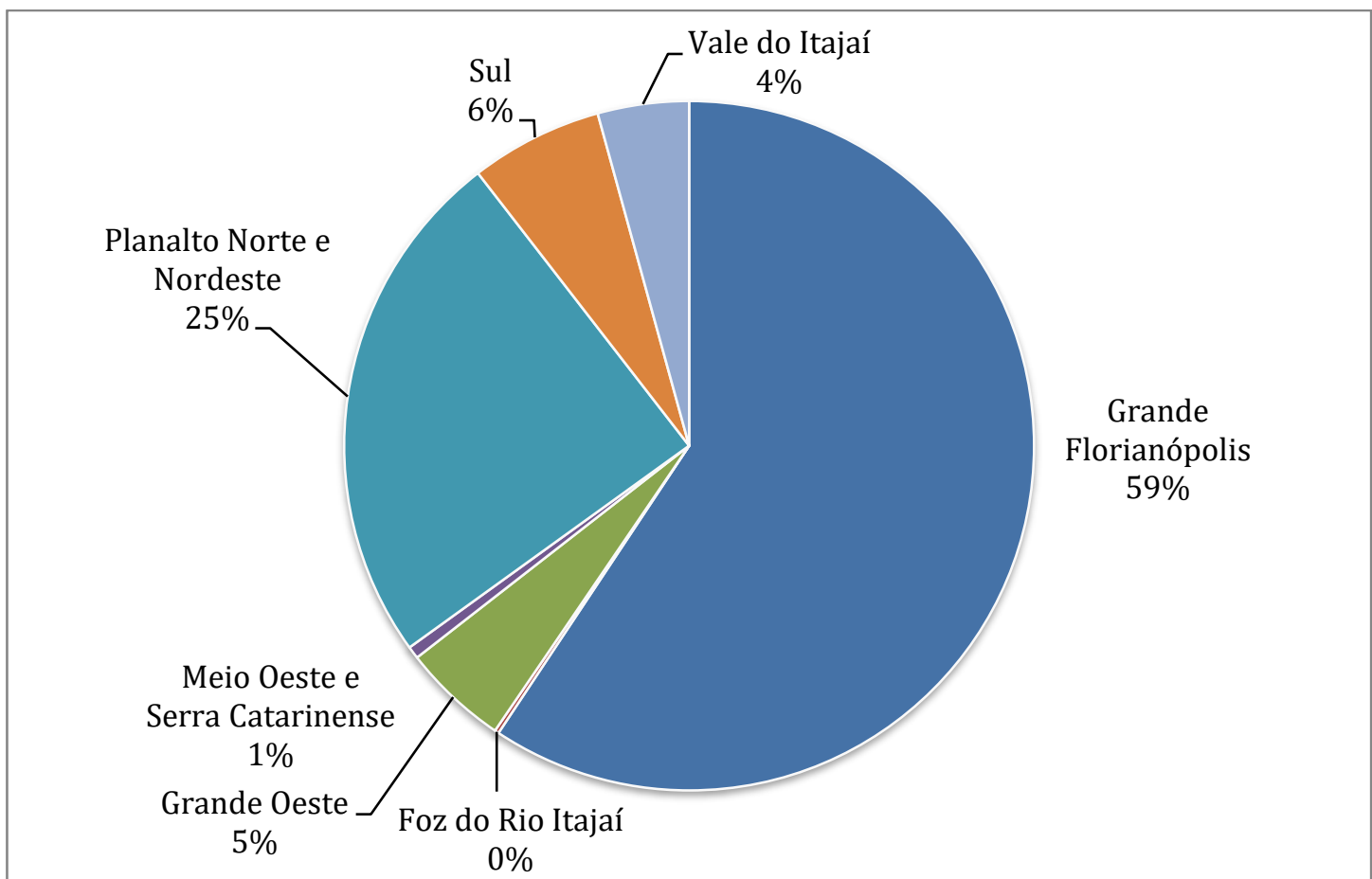

Fonte: INCA, 2021. Elaboração: FRIESTINO, JKO; FARIA, RM; GUERRA, P.H.A.; FRANCISCO, P.M.S.B, 2021.

A partir da análise da ocorrência de atendimentos em unidades hospitalares no estado de Santa Catarina, foram identificados 11 hospitais que atenderam casos de câncer em menores de 05 anos de idade no período estudado. A maioria dos estabelecimentos concentram-se na faixa litorânea, como pode ser observado na Figura 2. 
Figura 2. Distribuição dos hospitais habilitados para referência em oncologia, que apresentaram atendimentos de câncer em menores de 05 anos de idade, no período de 2009 a 2019 em Santa Catarina

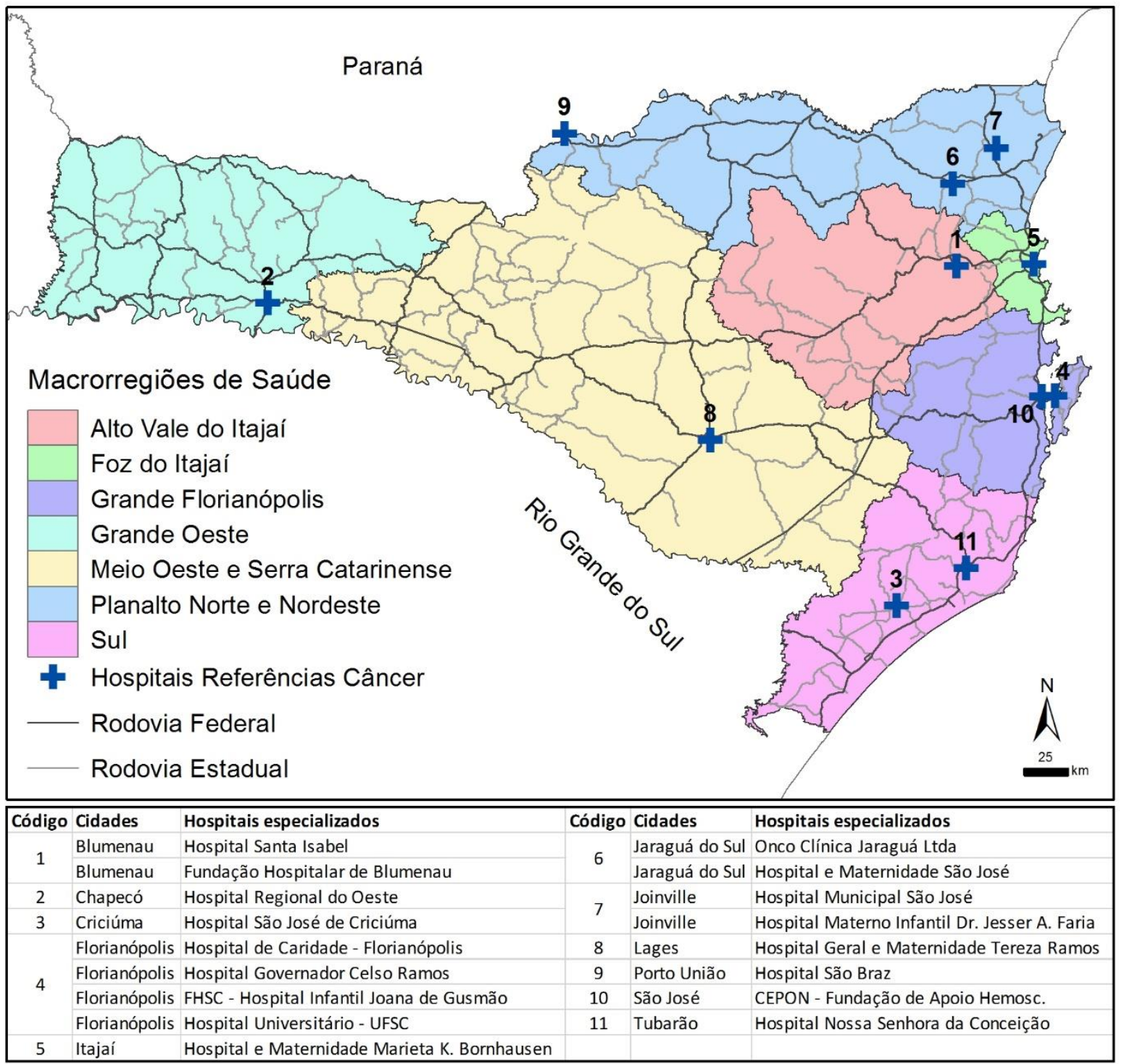

Fonte: INCA, 2021; DATASUS, 2021. Elaboração: FRIESTINO, JKO; FARIA, RM; GUERRA, P.H.A.; FRANCISCO, P.M.S.B, 2021.

Em relação ao número absoluto de internações entre 2009 e 2019 (Figura 3), observou-se que a macrorregião da Grande Florianópolis apresentou o total de 611 internações, seguida pela macrorregião Planalto Norte e Nordeste (252), Sul (64), Grande Oeste (51), Vale do Itajaí (44), Meio Oeste e Serra Catarinense (6) e Foz do Rio Itajaí (2), respectivamente. Considerando a distribuição anual absoluta dos registros no período, foi identificada forte correlação negativa e estatisticamente significativa $(r=-0,720 ; p=0,012)$ para a região do Meio Oeste e Serra Catarinense. Demais regiões não apresentaram correlação significativa. 
Figura 3. Registros hospitalares de câncer em menores de 05 anos de idade, segundo localização do hospital e macrorregião de saúde. Santa Catarina. 2009-2019

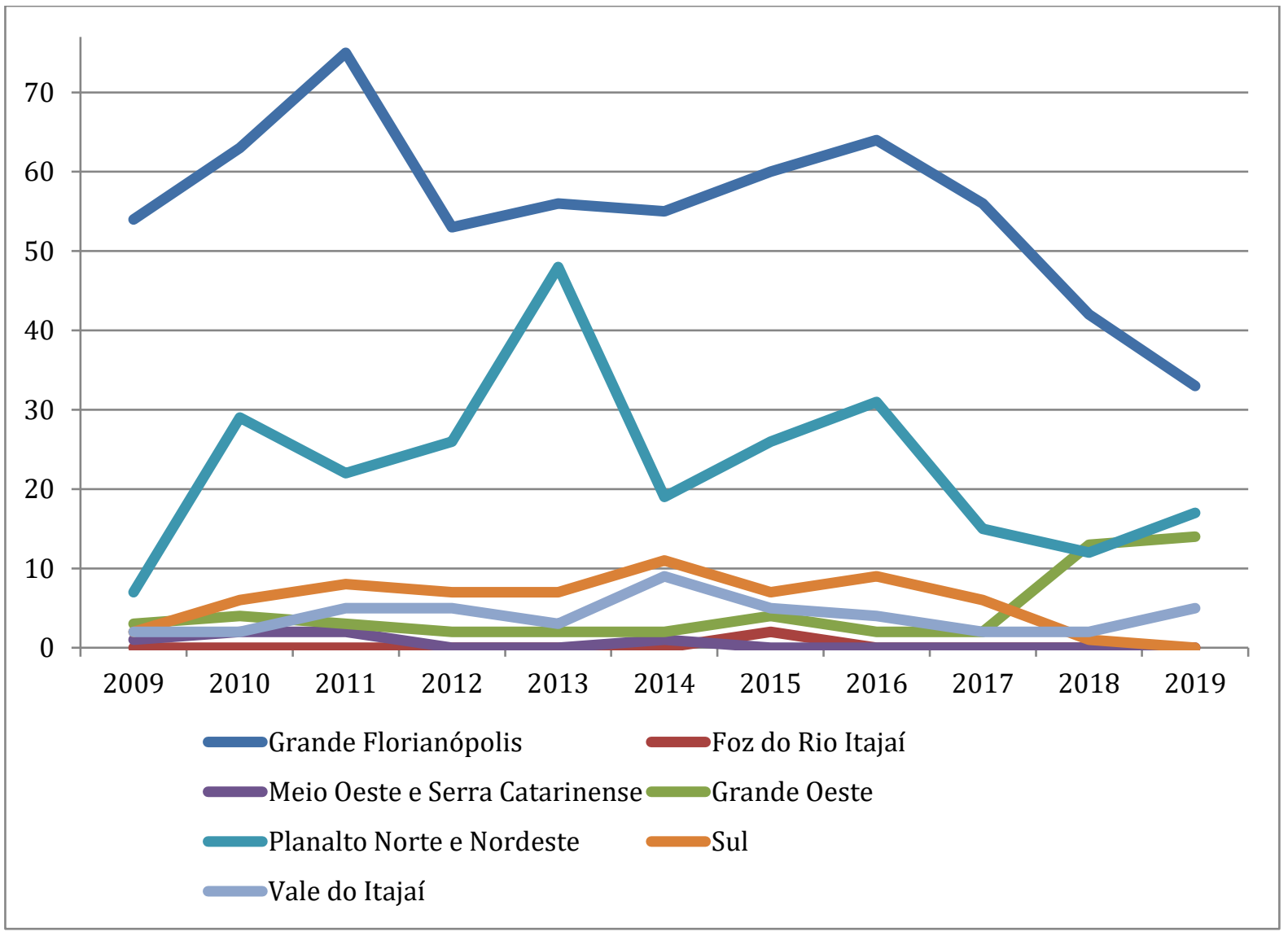

Fonte: INCA, 2021; DATASUS, 2021. Elaboração: FRIESTINO, JKO; FARIA, RM; GUERRA, P.H.A.; FRANCISCO, P.M.S.B, 2021.

\section{DISCUSSÃO}

Tendo como objetivo apresentar o perfil dos atendimentos hospitalares de câncer ocorridos na primeira infância no estado Santa Catarina ao longo de uma década, o presente estudo indicou a interiorização dos atendimentos ao longo dos anos, facilitando o acesso de crianças com câncer ao Sistema Único de Saúde.

A organização dos atendimentos hospitalares para a área de oncologia em Santa Catarina é norteada pelo Plano Diretor de Regionalização. Esse documento propõe a divisão do estado em 07 macrorregiões, com o objetivo de garantir o acesso ao conjunto de ações e serviços de saúde, de acordo com as necessidades da população (SANTA CATARINA, 2018).

Os atendimentos oncológicos são realizados, prioritariamente por estabelecimentos de saúde especializados, denominados: Centros de Assistência de Alta Complexidade em Oncologia (CACON), as Unidades de Assistência de Alta Complexidade em Oncologia (UNACON), sendo que, os demais hospitais devem atuar como suporte. As UNACON são unidades que tratam neoplasias de maior incidência, podendo ou não ofertar serviços de radioterapia, enquanto que CACON ofertam serviços especializados de maneira integral, incluindo radioterapia. Ambos os serviços direcionam-se para atuar no diagnóstico, estadiamento e tratamento, de acordo com a Política Nacional de Prevenção do Câncer, que trata dos parâmetros para organização, planejamento, controle, como também habilitação dos estabelecimentos no âmbito do SUS (BRASIL, 2013; BRASIL, 2014).

Considerando a complexidade no manejo do câncer, os estabelecimentos de saúde habilitados como CACON ou UNACON são distribuídos de acordo com a existência de integração da rede local à macrorregião de atenção à saúde. Além disso, o número de habilitações deve ser calculada para, no mínimo, cada 1.000 casos novos anuais, excluindo câncer não melanoma (BRASIL, 2019). O estado

\begin{tabular}{llllll}
\hline Hygeia & Uberlândia - MG $\quad$ Edição especial: X GeoSaude & Fev./2022 & p. 110-119 Página 115
\end{tabular}


de Santa Catarina possui 02 UNACON exclusiva de Oncologia pediátrica, estas situam-se na cidade de Florianópolis e Joinville, correspondentes às macrorregiões da Grande Florianópolis e Planalto Norte Nordeste respectiva.

As macrorregiões da Grande Florianópolis e Planalto Norte e Nordeste são as mais populosas, e concentram grande parte dos serviços de alta complexidade em oncologia (LOVATTO, et al, 2020). Em contrapartida, encontra-se a macrorregião do Grande Oeste como sendo a mais distante da capital, e consequentemente, dos centros especializados para o tratamento de câncer. Assim como o plano de regionalização é revisto periodicamente, a atenção oncológica também passa por mudanças, seguindo os pactuações das Comissões de Intergestores Bipartite e Tripartite, os quais resultam Termos de Garantia de Acesso (TCGA), que estabelecem prioridades no atendimento, buscando ordenar os serviços de forma equitativa, distribuindo as demandas nos diferentes serviços (SANTA CATARINA, 2018).

De acordo com Silva, et al (2020), a rede de atenção oncológica, em especial, à atenção pediátrica necessita ser fortalecida, pois ainda existem fragilidades relacionadas à tomada de decisões, principalmente no que diz respeito ao atendimento das demandas regionais, que por vezes resultam em grandes deslocamentos aos grandes centros em busca de tratamento, sendo esta uma situação que pode ser caótica para a criança e familiares.

Um estudo sobre câncer infantojuvenil no estado do Rio Grande do Sul, realizado por Algayer et al. (2020), apontou que nos últimos 20 anos as internações por câncer mais prevalentes ocorreram entre os menores de 4 anos, destacando-se um aumento nos últimos cinco anos, para faixa etária menor de 4 anos. A morbidade pode ser um indicador considerável para analisar as condições de saúde na primeira infância, pois conforme já mencionado, embora a ocorrência de câncer para essa faixa etária seja considerada rara, mesmo após o diagnóstico, a criança e sua família por vezes transitam em diversos cenários de atenção, além de percorrerem, às vezes, muitos quilômetros em busca de tratamentos especializados.

O percurso terapêutico percorrido para subsidiar o tratamento, estadiamento e/ou acompanhamento muitas vezes resulta na necessidade em traçar longos trajetos devido ao distanciamento das residências das crianças aos locais de referência. Além disso, durante o tratamento ou por decorrência deste, os pais apresentam diversas dificuldades, incluindo financeiras para custear além das viagens, até mesmo estadias nos grandes centros. (ALGAYER et al., 2020; GRABOIS; OLIVEIRA; CARVALHO, 2013). Vale lembrar que, os protocolos de quimioterapia e radioterapia indicam a necessidade de comparecimento ao serviço especializado várias vezes por semana, o que pode ser um fator de maior dificuldade para pacientes que residem há mais de $100 \mathrm{~km}$ do serviço de saúde (GRABOIS; OLIVEIRA; CARVALHO, 2013).

Embora a região Sul e Sudeste do Brasil concentrem a maioria dos serviços especializados (GRABOIS; OLIVEIRA; CARVALHO, 2013), notamos em nosso estudo que no estado de Santa Catarina existe uma concentração dos serviços nas macrorregiões dos grandes centros, incluindo a capital Florianópolis e a cidade mais populosa do estado que é Joinville. Em contraponto, a macrorregião do Grande Oeste, que possui como sede a cidade de Chapecó, encontra-se distante da capital cerca de $600 \mathrm{~km}$, tendo como principal acesso a rodovia SC 282. Em contraponto, ao longo dos dez anos estudados, esta macrorregião cada vez menos tem sido referenciada para outros centros de tratamento.

Com isso, a saúde infantil deve ser colocada em pauta, incluindo os indicadores de acesso aos serviços de saúde. Segundo Grabois, Oliveira e Carvalho (2013), a medida fundamental é garantir o acesso aos centros especializados, com equipamentos de suporte social e com a instauração de uma rede de referência que possibilite a realização de tratamento adequando, e consequentemente, melhores taxas de sobrevivência.

Em geral, a distribuição espacial dos serviços de saúde é feita segundo uma racionalidade baseada na economia de escala, ou seja, a depender da oferta e demanda um tal serviço pode ser economicamente adequado ou não (MENDES, 2011). A resolutividade dos serviços também depende de otimização, e, naturalmente, por essa questão, os serviços de saúde estarão concentrados nos grandes centros urbanos. Entretanto, se for considerada apenas a natureza matemática dessa economia, ou seja, como uma equação formulada pela oferta-demanda, sem consideração às desigualdades territoriais, como também aos desafios das fluidez no território, os problemas do acesso e uso serão intensificados, com o possível aumento das iniquidades assistenciais.

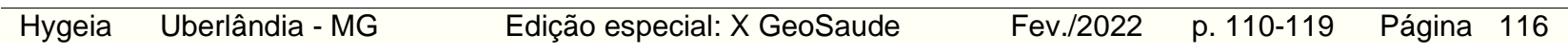


Os dados apresentados permitem inferir duas questões importantes, de maneira especial em relação à macrorregião do Grande Oeste (com sede em Chapecó). A primeira é a desconcentração espacial da oferta, seguida do aumento dos atendimentos no interior do estado. A segunda é a redução desta oferta nas áreas centrais, seguida da redução dos atendimentos no período avaliado. Portanto, isso indica uma mudança na estrutura espacial destes atendimentos, com repercussões territoriais e sociais importantes. Entre elas, a mudança na relação oferta-demanda, com o possível aumento populacional no Oeste do estado, que é algo que só poderemos saber com os dados do já atrasado Censo de 2020. De toda forma, o aumento dos atendimentos na região de Chapecó demonstra mudanças na geografia dos serviços de atendimento ao câncer na primeira infância.

Por outro lado, devemos acentuar o curioso fato de que a região mais extensa do estado (Meio Oeste/Serra Catarinense, com sede em Lages) e uma das menos populosas (com a menor densidade demográfica) teve redução no número de internações. Desde 2015 a região não registrou internações, - que leva a concluir que não houve oferta do serviço, uma vez que, a considerar a natureza do indicador, a demanda é sempre crescente. Neste sentido, não se pode aplicar os princípios da economia de escala sem considerar as dificuldades dos deslocamentos para tratamento numa região tão extensa.

Vale ressaltar que as características inerentes ao agravo do câncer, em especial aos que ocorrem na infância, são permeados por diversas admissões hospitalares, as quais constituem etapas necessárias e frequentes na trajetória com a doença, iniciando na fase diagnóstica, e seguido na etapa de tratamento, incluindo sintomas graves ou ainda em fase terminal (ANTONUZZO, et.al., 2017; NUMICO et.al., 2020). Com isso, deve-se considerar, aliás, os desafios para o deslocamento em relação às necessidades, ou seja, ao tratamento do câncer nesta faixa etária, envolvendo não apenas o sujeito, mas a família como um todo. Naturalmente, a distribuição espacial das internações por câncer obedece o critério da oferta, sendo tanto mais volumosas quanto maior for esta oferta. Neste sentido, o que se observa é a necessidade de ampliar tal oferta no interior do estado, levando-se em consideração não apenas a economia de escala, mas os perfis sociais e territoriais do estado catarinense.

Como limitação do estudo citamos o uso de dados secundários, isso porque, não foi possível realizar um controle nos processos de subnotificação ou má qualidade dos registros.

\section{CONSIDERAÇÕES FINAIS}

Com a organização da rede de assistência ao câncer pediátrico, houve uma interiorização dos atendimentos ao longo dos anos, beneficiando regiões que se encontram mais distantes da maioria dos serviços de alta complexidade em oncologia pediátrica, possibilitando assim, melhorias nas condições de acesso aos serviços. O estudo teve como base informações disponíveis ao público e, por tratar-se de uma apresentação regional, recomenda-se que sejam realizadas trabalhos futuros em outros cenários, visando a discussão e melhorias para a saúde infantil.

\section{REFERÊNCIAS}

ALMEIDA, W. S. da; SZWARCWALD, C. L. Mortalidade infantil e acesso geográfico ao parto nos municípios brasileiros. Rev. Saúde Pública, v. 46, n. 1; p. 68-76, 2012. DOI: https://doi.org/10.1590/S0034-89102012005000003

ALGAYER, L. P. et al. Tendência Temporal de Internações por Diagnóstico Oncológico em Crianças e Adolescentes. Rev. Bras. Cancerologia, v.66, n. 4, p. e-141010, 2020. DOI: https://doi.org/10.32635/2176-9745.RBC.2020v66n4.1010

ANTONUZZO, A. et al. Impact of a supportive care service for cancer outpatients: management and reduction of hospitalizations: preliminary results of an integrated model of care. Support Care Cancer, v. 25, n. 1, p. 209-212. DOI: 10.1007/s00520-016-3403-z. Epub 2016 Sep 11, jan. 2017

BLACK, M.M. et al. Early childhood development coming of age: science through the life course. Lancet. v. 389, n. 10064, p. 77-90, 2017. DOl: https://doi.org/10.1016/S0140-6736(16)31389-7.

BRASIL. Ministério da Saúde. Diretrizes Operacionais dos Pactos pela Vida, em Defesa do SUS e de Gestão. Brasília: Ministério da Saúde, 2006.

$\begin{array}{lllll}\text { Hygeia } & \text { Uberlândia - MG } \quad \text { Edição especial: X GeoSaude } & \text { Fev./2022 } & \text { p. 110-119 } & \text { Página } 117\end{array}$


BRASIL. Ministério da Saúde. Portaria № 874, de 16 de maio de 2013. Institui a Política Nacional para a Prevenção e Controle do Câncer na Rede de Atenção à Saúde das Pessoas com Doenças Crônicas no âmbito do Sistema Único de Saúde (SUS). 2013. Disponível em:

http://bvsms.saude.gov.br/bvs/saudelegis/gm/2013/prt0874 1605 2013.html. Acesso em: 30 nov 2021.

BRASIL. Ministério da Saúde. Portaria № 140, de 27 de fevereiro de 2014. Redefine os critérios e parâmetros para organização, planejamento, monitoramento, controle e avaliação dos estabelecimentos de saúde habilitados na atenção especializada em oncologia e define as condições estruturais, de funcionamento e de recursos humanos para a habilitação destes estabelecimentos no âmbito do Sistema Único de Saúde (SUS). 2014. Disponível em:

http://bvsms.saude.gov.br/bvs/saudelegis/sas/2014/prt0140 2702 2014.html. Acesso em: 30 nov 2021.

BRASIL. Ministério da Saúde. Portaria SAES/MS, № 1399, de 17 de dezembro de 2019. Redefine os critérios e parâmetros referenciais para a habilitação de estabelecimentos de saúde na alta complexidade em oncologia no âmbito do SUS. 2019. Disponível em:

https://www.inca.gov.br/sites/ufu.sti.inca.local/files//media/document//portaria 1399 17dez2019.pdf. Acesso em: 30 nov 2021.

DATASUS. Departamento de Informática do SUS. Cadastro Nacional de Estabelecimentos de Saúde. 2021. Disponível em: http://cnes.datasus.gov.br/pages/consultas.jsp. Acesso em: 01 dez 2021.

DIAS, B. A. S. et al. Análise espacial dos óbitos infantis evitáveis no Espírito Santo, Brasil, 2006-2013. Epidemiol. Serv. Saúde, v. 28, n. 3, p. 1-11, 2019. DOI: https://doi.org/10.5123/S167949742019000300001.

DORNELLES, R.; AREOSA, S.C. A governança como instrumento de ampliação da regionalização da saúde no Rio Grande do Sul. Sau. \& Transf. Soc. v. 11, n. 2, p. 27-46, 2020. DOI: http://stat.entrever.incubadora.ufsc.br/index.php/saudeetransformacao/article/view/5557/5675.

FARIA, R.M. A territorialização da atenção primária à saúde no Sistema Único de Saúde e a construção de uma perspectiva de adequação dos serviços aos perfis do território. Hygeia, v. 9, n. 16, p. $131-147,2013$.

FARIA, R. M.; SANTANA, P. Regional inequalities in infant mortality and primary health care in the State of Minas Gerais, Brazil. Space Populations and Societies, v. 2, n. 3, p. 1-14, 2015. https://doi.org/10.4000/eps.5852

GOLDANI, M. Z. et al. Infant mortality rates according to socioeconomic status in a Brazilian city. Revista de Saúde Pública, v. 35, n. 3, p. 256-261, 2001. https://doi.org/10.1590/S0034$\underline{89102001000300007}$

GRABOIS, M.F.; OLIVEIRA, E.X.G; CARVALHO, M.S. Assistência ao câncer entre crianças e adolescentes: mapeamento dos fluxos origem-destino no Brasil. Rev Saúde Pública, v. 47, n. 2, p. 368-78, 2013. DOI: https://doi.org/10.1590/S0034-8910.2013047004305.

INCA. Instituto Nacional do Câncer José Alencar Gomes da Silva. Integrador RHC. Disponível em: https://irhc.inca.gov.br/RHCNet/visualizaTabNetExterno.action. Acesso em: 30 nov 2021.

LOVATTO, M. V. P. et al. Análise Documental em Relação ao Fluxo de Assistência do Câncer Infantojuvenil em Santa Catarina. In: Jane Kelly Oliveira Friestino; Maíra Rossetto; Vander Monteiro da Conceição. (Org.). Câncer infanto-juvenil : pesquisas epidemiológicas e organização dos serviços em Santa Catarina. 1ed.Goiânia: Editora Espaço Acadêmico, 2020, p. 21-32.

MENDES, E. V. Redes de atenção à saúde. Brasília: OPAS, 2011.

NUMICO, G., et al. The hospital care of patients with cancer: a retrospective analysis of the characteristics of their hospital stay in comparison with other medical conditions. Eur J Cancer. v. 1399, p. 99-106, nov, 2020. https://doi.org/10.1016/j.ejca.2020.08.023

PIRES, L.J.A. O Câncer Infantojuvenil nas Políticas Públicas no Estado do Rio de Janeiro, 2013-2021. Rev. bras. cancerol. v. 64, n. 3, p. 397-400, 2018. DOI: https://doi.org/10.32635/2176-

9745.RBC.2018v64n3.46. https://doi.org/10.32635/2176-9745.RBC.2018v64n3.46

$\begin{array}{llllll}\text { Hygeia Uberlândia - MG } \quad \text { Edição especial: X GeoSaude } & \text { Fev./2022 } & \text { p. 110-119 } & \text { Página } 118\end{array}$


SANTA CATARINA. Secretaria Estadual de Saúde. Plano Diretor de Regionalização. Florianópolis. 2018. Disponível em: http://www.saude.sc.gov.br/index.php/informacoes-gerais-documentos/planejamento-em-saude/instrumentos-de-gestao-estadual/plano-diretor-de-regionalizacao/14617-planodiretor-de-regionalizacao-2018/file. Acesso em: 30 nov. 2021.

SILVA, B.Q.; et al.. Distribuição de óbitos de câncer infanto-juvenil nas regionais de saúde do estado do Rio de Janeiro. Rev. pesqui. cuid. fundam. v.12, p. 890-896, 2020. DOI:

http://dx.doi.org/10.9789/2175-5361.rpcfo.v12.7916.

SZWARCWALD, C. L.; ANDRADE, C. T.; BASTOS, F. I. Income inequality, residential poverty clustering and infant mortality: a study in Rio de Janeiro, Brazil. Social Science \& Medicine, v. 55. n. 12, p. 2083-2092, 2002. https://doi.org/10.1016/S0277-9536(01)00353-7

UNICEF. United Nations Children's Fund. Early moments matter for every child. UNICEF: New York. 2017. Disponível em:

https://www.unicef.org/media/48886/file/UNICEF Early Moments Matter for Every Child-ENG.pdf. Acesso em: 01 dez 2021. 